\title{
Improved error bounds for the erasure/list scheme: the binary and spherical cases
}

\author{
Alexander Barg *
}

\begin{abstract}
We derive improved bounds on the error and erasure rate for spherical codes and for binary linear codes under Forney's erasure/list decoding scheme and prove some related results.
\end{abstract}

Keywords: distance distribution, erasure/list decoding, error exponent, spherical codes.

\section{Introduction}

The subject of error bounds for various decoding schemes has been a central topic in information theory in its first decades. With the success of turbo codes and other iterative decoding schemes this subject again became the focus of continued attention through the last decade. In the early days the major effort in deriving error bounds went into establishing the best attainable error exponents (for instance, Shannon's reliability function of channels). This approach is reflected in most textbooks on information theory that deal with this subject [9, 10, 19, 2, 5]. Lately the attention has shifted from considering average properties of code ensembles to bounding the error probability of decoding of a particular code whose distance distribution is known or can be estimated. Focusing on a particular code instead of an ensemble of codes makes it possible to analyze the error probability by a geometric approach rather than Chernoff bounds. These studies gained momentum after influential research of G. Poltyrev in [13, 14]; see [16] and references therein.

It is interesting to note that Shannon [17] also relied on a geometric derivation in his paper on the error bounds for spherical codes and the Gaussian channel. The starting point of the present research was an attempt to derive Shannon's results via the distance distribution of the code (recall that about the original derivation the author wrote: "It might be said that the algebra involved is in several places unusually tedious"). It turns out that in this way the results of [17] can be obtained by a simpler, more intuitive argument. To add a new element to this study, we consider a version of Forney's erasure/list decoding scheme [7], [8]. To define it, let $C$ be a code in a metric (observation) space $X$ with the metric $\mathrm{d}(\cdot, \cdot)$ and let $t \geq 0$. The decoding function $\psi_{t}$ is defined as follows: $\psi_{t}(\mathbf{y})=\mathbf{x}$ if for all code vectors $\mathbf{x}^{\prime} \neq \mathbf{x}$ the distance $\mathrm{d}\left(\mathbf{x}^{\prime}, \mathbf{y}\right)-\mathrm{d}(\mathbf{x}, \mathbf{y}) \geq 2 t$. For all other points in $X$ the decoding result is undefined and will be called erasure below.

We will be interested in the best attainable exponents of error and erasure probabilities, denoted $E_{e}$ and $E_{x}$ below. Error bounds for this decoding for general discrete memoryless channels were derived in [7], [8]. In particular, they imply bounds on $E_{e}$ and $E_{x}$ for unrestricted codes in the Hamming space used over a

\footnotetext{
${ }^{*}$ University of Maryland, College Park, MD 20742, e-mail: abarg@ ieee.org. Supported in part by NSF grant CCR-0310961.
} 
binary symmetric channel. The case of linear codes was addressed by Blokh and Zyablov [3]. Error bounds for this decoding method in the case of spherical codes are not available in the literature.

The text is organized as follows. In Sect. 2 we address the technically easier and more familiar case of binary linear codes. The main goal of this part is to develop geometric intuition in a more familiar situation and then to rely on it in a more difficult case of spherical codes. However, as a byproduct, we obtain an improvement of the bounds of [3] on $E_{e}$ and $E_{x}$. Moreover, the method we use is arguably easier to understand than the results in [3].

In Sect. 3 we consider the case of bounded distance decoding and some other related questions.

In the second part we study spherical codes. For the Gaussian channel we obtain a pair of bounds that specifies the trade-off between the error and erasure events. For $t=0$ the bounds reduce to Shannon's lower bound on the error exponent of maximum likelihood decoding. In our calculations we rely on the distance distribution of codes. Note that Shannon's derivation [17], although geometric in nature, takes a somewhat different route, performing averaging of the error probability over the choice of codes. This method is not the best known for low noise because average codes contain small distances, so expurgation of the code ensemble is needed to obtain a good bound for low rates. In contrast, we begin with choosing codes with large minimum distance and obtain the complete result by a single argument. Since we operate in terms of the distance distribution, we will obtain some new insights into the decoding geometry of spherical codes in the course of our derivation. We also outline a derivation of Shannon's error bounds [17] by an approach which is arguably simpler than both the original proof and Gallager's proof in [10]. The proof method considered exhibits a close analogy between spherical codes and codes in $\{0,1\}^{n}$ if one makes allowance for some peculiarities of discrete geometry.

We also derive error bounds for bounded distance decoding of spherical codes. This problem was mentioned in [20], however the focus of that paper is on different questions. In particular, we address the question of the probability of undetected error with spherical codes, in the sense specified in the main text, and establish the asymptotic behavior of this quantity.

\section{The binary case}

Let $X=\{0,1\}^{n}$ be the binary Hamming space with distance $\mathrm{d}(\cdot, \cdot)$. We consider linear codes $C \subset X$ of rate $R=n^{-1} \log _{2}|C|$ used over a binary symmetric channel with crossover probability $p \in(0,1 / 2)$.

For a code $C \subset X$ consider a decoding mapping mapping $\psi_{t}: X \rightarrow C$ defined as follows: $\psi_{t}(\mathbf{x})=\mathbf{c}$ if for all code vectors $\mathbf{c}^{\prime} \neq \mathbf{c}$ the distance $\mathrm{d}\left(\mathbf{c}^{\prime}, \mathbf{x}\right)-\mathrm{d}(\mathbf{c}, \mathbf{x}) \geq 2 t$ for some nonnegative integer $t=\tau n$. For all other points in $X$ the decoding result is undefined, and will be called erasure below. For the case of complete decoding we write $\psi$ instead of $\psi_{0}$.

Let us introduce notation. Denote by $A_{w}, w=0,1, \ldots, n$ the weight distribution of $C$. For a code of minimum distance $d$ we have $A_{0}=1, A_{1}=\cdots=A_{d-1}=0$. Let us introduce the weight profile of the code: for $\omega=w / n, w=0,1, \ldots, n$ let

$$
\alpha(\omega)=\frac{1}{n} \log _{2} A_{w},
$$

where $\log 0=-\infty$.

$$
\begin{aligned}
& \text { Let } \mathcal{A}_{0}=1, \mathcal{A}_{w}=\left\lfloor\left(\begin{array}{c}
n \\
w
\end{array}\right) 2^{-n(1-R)}\right\rfloor, w=1, \ldots, n, \\
& \qquad(x, y)=-x \log _{2} y-(1-x) \log _{2}(1-y),
\end{aligned}
$$


$h(x):=T(x, x), D(x \| y):=T(x, y)-h(x)$. Throughout the rest of the text $\delta_{\mathrm{GV}}(R)=h^{-1}(1-R)$ is the relative Gilbert-Varshamov $(\mathrm{GV})$ distance and $d=d_{\mathrm{GV}}=\left\lfloor\delta_{\mathrm{GV}}(R) n\right\rfloor$. Let $E_{0}(R, p)$ be the Gallager bound on the reliability function of the channel [9, pp.34-36]:

$$
E_{0}(R, p)= \begin{cases}-\delta_{\mathrm{GV}} \log _{2} 2 \sqrt{p(1-p)} & 0 \leq R \leq R_{e} \\ D\left(\rho_{0} \| p\right)+R_{c}-R & R_{e} \leq R \leq R_{c} \\ D\left(\delta_{\mathrm{GV}}(R) \| p\right), & R_{c} \leq R \leq 1-h(p),\end{cases}
$$

where

$$
\begin{gathered}
\rho_{0}=\frac{\sqrt{p}}{\sqrt{p}+\sqrt{1-p}}, \quad \omega_{0}=2 \rho_{0}\left(1-\rho_{0}\right), \\
R_{e}=1-h\left(\omega_{0}\right), \quad R_{c}=1-h\left(\rho_{0}\right) .
\end{gathered}
$$

Denote by $S_{r}(0)$ a ball of radius $r$ in $X$ with center at 0 and by

$$
p_{i, j}^{k}=\mid\{\mathbf{z} \in X: \mathrm{d}(\mathbf{z}, \mathbf{x})=i, \mathrm{~d}(\mathbf{z}, \mathbf{y})=j ; \mathrm{d}(\mathbf{x}, \mathbf{y})=k \|
$$

the number of triangles in $X$ with a fixed side of length $k$. Let $\nu=\log _{2}((1-p) / p)$.

For unrestricted codes various lower bounds on the exponents $E_{e}, E_{x}$ were given in Forney [8]. For linear binary codes the following theorem was proved by Blokh and Zyablov.

Theorem 1 [3] For $0 \leq R<R_{c}$

$$
\begin{aligned}
& E_{e}(R, p, \tau) \geq E_{0}(R, p)+\nu \tau \\
& E_{x}(R, p, \tau) \geq E_{0}(R, p)-\nu \tau
\end{aligned}
$$

For $R \geq R_{c}$

$$
\begin{aligned}
& E_{e}(R, p, \tau) \geq E_{0}(R, p)+\left.2 \tau D^{\prime}(\delta \| p)\right|_{\delta=\delta_{\mathrm{GV}}(R)} \\
& E_{x}(R, p, \tau) \geq E_{0}(R, p)-\left.2 \tau D^{\prime}(\delta \| p)\right|_{\delta=\delta_{\mathrm{GV}}(R)} .
\end{aligned}
$$

Note that the case $t=0$ corresponds to maximum likelihood decoding, and the bound on $E_{e}$ turns into $E_{0}$. Erasure rate in this case is of course zero though (3), (5) give a positive value, because by the nature of the argument the erasure probability $P_{x}$ is estimated by the sum $P_{e}+P_{x}$.

REMARK 1: Note also that by (3), the exponent $E_{x}=0$ for rates in the range close to the channel capacity. In this range the value of the undetected error exponent $E_{e}$ can be claimed arbitrarily large if we modify the decoding function to claim an erasure for all transmissions. Thus, in effect Theorem 1 contains a nontrivial claim only for those values of the code rate $R$ for which $E_{x}>0$, i.e., for which the right-hand side of (5) is positive. Of course, even when $E_{x}=0$ i.e. decoding results in erasures in almost all transmissions, it is still useful to know how often we will run into an undetected error.

The aim of this section is to derive lower bounds on the exponents which are better than the estimates (2)-(5) for most values of $R>0$. To state the results we need the following definitions: $u=p(1-p)$,

$$
\begin{aligned}
\rho_{0}^{ \pm} & =\frac{\sqrt{u+\tau^{2}(1-2 p)^{2}}-p(1 \pm 2 \tau) \pm \tau}{1-2 p} \\
\omega_{0} & =2 \rho_{0}^{ \pm}\left(1-\rho_{0}^{ \pm}\right) \pm 2 \tau\left(1-2 \rho_{0}^{ \pm}\right) \\
& =2 \frac{\sqrt{u+\tau^{2}(1-4 u)}-2 u}{1-4 u}
\end{aligned}
$$




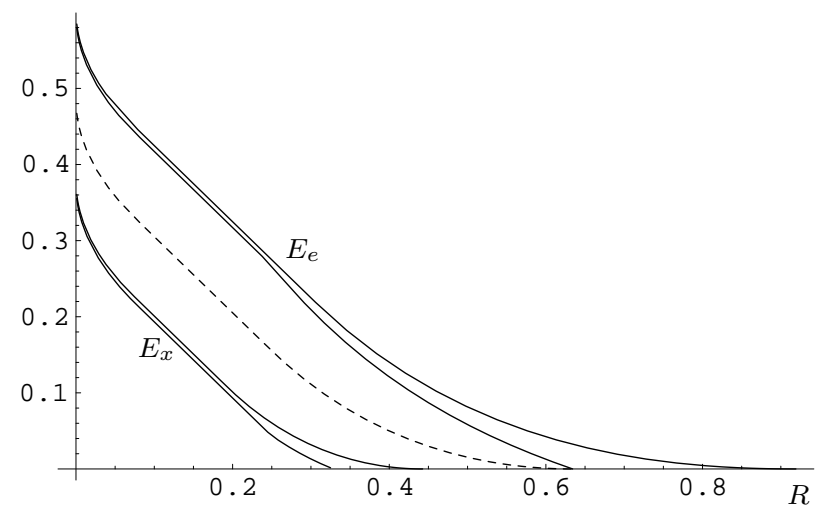

Figure 1: Error bounds from Theorems 1 and $2(p=0.07, \tau=0.03)$. In each pair the better bound is from Thm. The dashed line is the function $E_{0}(R, p)$.

$$
M_{ \pm}= \begin{cases}-\delta_{\mathrm{GV}}(R)\left(h\left(\frac{1}{2}+\frac{\tau}{\delta_{\mathrm{GV}}}\right)+\frac{1}{2} \log _{2} u\right) \pm \nu \tau & 0 \leq R \leq 1-h\left(\omega_{0}\right) \\ D\left(\rho_{0}^{ \pm} \| p\right)+1-R-h\left(\rho_{0}^{ \pm} \mp 2 \tau\right) & 1-h\left(\omega_{0}\right)<R \leq 1-h\left(\rho_{0}^{ \pm}\right) \\ D\left(\delta_{\mathrm{GV}} \pm 2 \tau \| p\right) & R>1-h\left(\rho_{0}^{ \pm}\right) .\end{cases}
$$

We then have the following result whose proof is given in the appendix.

Theorem 2 Let $R \geq 1-h(0.5-\tau)$, then the exponent of the undetected error is bounded below as

$$
E_{e}(R, p, \tau) \geq M_{+} \text {. }
$$

Let $R \geq 0, \tau \leq p / 2$, then the erasure exponent is bounded below as

$$
E_{x}(R, p, \tau) \geq M_{-} .
$$

Remark 1 applies to this theorem as well: the claim of the theorem is nontrivial for code rates below $1-h(p+2 \tau)$.

For $\tau=0$ the bounds also reduce to $E_{0}(R, p)$, as expected. However, they are strictly greater that the bounds of Theorem 1 For instance, for the case (c) this can be proved using the fact that $D(\delta \| p)$ is a $\cup$-convex increasing function of $\delta$ for $\delta>p$ :

$$
M_{+}=D\left(\delta_{\mathrm{GV}}(R)+2 \tau \| p\right)>D\left(\delta_{\mathrm{GV}}(R) \| p\right)+2 \tau D_{\delta}^{\prime}\left(\delta_{\mathrm{GV}}(R) \| p\right)
$$

It is also easy to establish similar inequalities in the other cases. Typified behavior of the bounds on $E(R, \rho, \tau)$ from Theorems 1 and 2 is shown in Fig. 1 These theorems and the other results in the binary case extend in a standard way to binary-input output-symmetric discrete memoryless channels and to the $q$-ary symmetric channel, $q \geq 2$.

REMARK 2: The conditions $R \geq 1-h(1 / 2-\tau)$ and $\tau \leq p / 2$ seem to make Theorem 2 sound more restrictive than Theorem 1 It is possible to remove these conditions and prove somewhat weaker bounds 
which will still improve upon Theorem 1 However, the first of the two conditions for small $\tau$ is not a substantial restriction of the range of codes rates: for instance, for $\tau=0.03$ the bound on $E_{e}$ is valid for all code rates $R \geq 0.0025$. Furthermore, it is often the case that the bounds (2)-(5) are void while Theorem 2 claims nontrivial results. For instance, for $(p, \tau)=(0.2,0.09)$ the bound 5 and hence the rest of Theorem 1 is trivial while Theorem 2 gives nontrivial exponential error bounds for small values of the code rate.

REMARK 3: As indicated above, Theorem 1 can be obtained by a small modification of the proof of the our result. Generally, Theorem 11 claims results weaker than those in (6)-(7) because the authors of [3] in their derivation relied on a suboptimal decision region.

REMARK 4: We can add some details on typical error events in the course of decoding. For instance, consider the error-only case. Let $\rho_{\text {typ }}$ be the relative weight of error vectors that lead to a decoding error, and let $\omega_{\text {typ }}$ be the relative weight of code vectors obtained as a result of incorrect decoding. From the proof it is clear that for the case (a), $\rho_{\mathrm{typ}}=\left(1-\delta_{\mathrm{GV}}\right) p+\delta_{\mathrm{GV}} / 2+\tau, \omega_{\mathrm{typ}}=\delta_{\mathrm{GV}}$. For the case (b), $\rho_{\text {typ }}=\rho_{0}, \omega_{\mathrm{typ}}=\omega_{0}$. Finally, for the case (c), $\rho_{\mathrm{typ}}=\delta_{\mathrm{GV}}, \omega_{\mathrm{typ}}=2 \delta_{\mathrm{GV}}\left(1-\delta_{\mathrm{GV}}\right)+2 \tau\left(1-2 \delta_{\mathrm{GV}}\right)$. A more detailed discussion of these results for $\tau=0$ is provided by [1].

REMARK 5: Note an alternative expression for the case (b) of $M_{ \pm}$

$$
M_{ \pm}=1-R-h\left(\omega_{0}\right)-\omega_{0} h\left(\frac{1}{2} \pm \frac{\tau}{\omega_{0}}\right)-\frac{\omega_{0}}{2} \log _{2} u \pm \nu \tau .
$$

We stress that the dependence of the bound on $\tau$ is essentially nonlinear, contrary to the closed-form bounds in [8],[3].

\section{Related results: The binary case}

1. Let us address the question of error bounds for a specific code under max-likelihood decoding. Let $C$ be a code with distance distribution $A_{w}=2^{n \alpha(\omega)}(\omega=w / n, 0 \leq w \leq n)$ and let

$$
K(C)=\max _{1 \leq w \leq n} \frac{A_{w}}{\max \left(1, \mathcal{A}_{w}\right)},
$$

$\kappa(C):=n^{-1} \log _{2} K(C)$. For simplicity only we put $\tau=0$. The following bound is straightforward.

Theorem 3

$$
n^{-1} \log \frac{1}{P_{d e}(C)} \geq \max \left(D, E_{0}(R, p)-\kappa(C)\right)-o(1)
$$

where

$$
D=-\max _{0<\omega \leq 1}\left(\alpha(\omega)+(\omega / 2) \log _{2}(4 u)\right)
$$

PROOF. Denote by $P_{e}(w)$ the error probability under the condition that the decoded vector is $w$ away from the transmitted one. Then

$$
P_{d e}(C) \leq \sum_{w=1}^{n} A_{w} P_{e}(w)
$$

Taking logarithms and switching to exponents, we obtain the first part of the claim. The second part is equally obvious because

$$
\begin{aligned}
P_{d e}(C) & \leq \sum_{w=1}^{n} A_{w} P_{e}(w) \leq 2^{-n(1-R)} K(C) \sum_{w=d}^{n}\left(\begin{array}{l}
n \\
w
\end{array}\right) P_{e}(w) \\
& \leq 2^{n\left(\kappa(C)-E_{0}(R, p)-o(1)\right)} .
\end{aligned}
$$


Note that if $R_{e} \leq R<R+\kappa(C) \leq R_{c}$, then $E_{0}(R, p)$ is given by a linear function with slope -1 , and we can write $E_{0}(R, p)-\kappa(C)=E_{0}(R+\kappa(C), p)$. The second part of the bound under the maximum in (8) is the main result of [18], see Theorem 1 of that paper]. The above proof is a shorter way to obtain it.

Note also that for low $R$ bound $D$ on the error rate of $C$ can be better (and is never worse) than the second part of 8 . This is due to the fact that in $D$ we maximize the product of the weight profile and the pairwise error probability, while in the second bound the maximization of these two terms is separate.

2. Consider the decoding procedure $\tilde{\psi}_{t}$ under which $\tilde{\psi}_{t}(\mathbf{x})=\mathbf{c}$ if $\mathrm{d}(\mathbf{c}, \mathbf{x}) \leq t$ and $\tilde{\psi}_{t}(\mathbf{x})$ undefined if such a code vector does not exist. In this case the calculation of the error exponent is cumbersome, and depends on the relation between $p$ and $t$. One particular case is easy to analyze.

Proposition 4 Let $\mathcal{C}$ be a linear binary code with weight distribution $\mathcal{A}_{i}, i=0, \ldots, n$. Suppose that for every $d \leq w \leq n$ the maximum of

$$
\sum_{i=\max (\lceil w / 2\rceil, w-t)}^{n} \sum_{\ell=0}^{t}\left(\begin{array}{c}
w \\
i
\end{array}\right)\left(\begin{array}{c}
n-w \\
\ell
\end{array}\right) p^{i+\ell}(1-p)^{n-i-\ell}
$$

is attained for $\ell=0, i=w-t$. Then $-n^{-1} \log P_{d e}(C) \geq \tilde{E}_{e}(R, p, \tau)-o(1)$, where

$$
\tilde{E}_{e}(R, p, \tau)= \begin{cases}-\delta h(\tau / \delta)-T(\delta-\tau, p), & 0 \leq R \leq 1-h(p+\tau(1-p)) \\ 1-R-h(\tau)-\tau \log _{2}(1-p) & 1-h(p+\tau(1-p)) \leq R\end{cases}
$$

Proof. (outline) We have

$$
P_{d e}(C) \leq(t+1)^{2} 2^{-n(1-R)} \sum_{w=d}^{n}\left(\begin{array}{c}
n \\
w
\end{array}\right)\left(\begin{array}{c}
w \\
w-t
\end{array}\right) p^{w-t}(1-p)^{n-w+t} .
$$

In the sum on $w$ the summation term is maximized for $w \approx n(p+\tau(1-p))$. The exponent in question is obtained by computing the logarithms and depends on the sign of $p+\tau(1-p)-\delta$. For $p+\tau(1-p) \leq \delta$ the dominating term is the one with $w=d$. Upon simplification we obtain the first case of the claimed bound. Otherwise the maximum is within the summation range. Taking logarithms, substituting $\omega=p+\tau(1-p)$ and simplifying, we obtain the second case.

In particular, let $t=0$, which corresponds to the case of pure error detection. Then $\tilde{E}_{e}(R, p, \tau)$ reduces to the well-known lower bound on the exponent of undetected error [11].

\section{Spherical codes}

In this section we address the problem of error bounds for erasure decoding for the case of spherical codes. We assume transmission over a Gaussian channel with signal-to-noise ratio $A$. Let $S^{n-1}(r, \mathbf{x})$ be the sphere in $\mathbb{R}^{n}$ of radius $r$ with center at $\mathbf{x}$. We will write $S^{n-1}(r)$ for $S^{n-1}(r, \mathbf{0})$.

Let $X=S^{n-1}(\sqrt{A n})$ and let $\mathbf{y}_{1}, \mathbf{y}_{2} \in X$ be two vectors. One way to measure the distance between them is by the angle $\angle\left(\mathbf{y}_{1}, \mathbf{y}_{2}\right)$, and we will write $\mathrm{d}\left(\mathbf{y}_{1}, \mathbf{y}_{2}\right)=\phi$ if this angle equals $\phi$. The distance of a code $C \subset X$ is defined in the usual way as the minimum pairwise distance in $C$. For a given vector $\mathbf{x}$ if $\mathbf{y}=\mathbf{x}+\mathbf{z}$ and $\mathrm{d}(\mathbf{y}, \mathbf{x})=\phi$, we will say that $\mathbf{z}$ has weight $\phi$. 
For a code $C \in X$ let $M$ be its size, $R=n^{-1} \ln M$ its rate and $\theta=\theta(C)$ its distance. We also define the distance distribution of $C$ as follows:

$$
B(s, t)=M^{-1}\left|\left\{\mathbf{x}, \mathbf{x}^{\prime} \in C: s \leq \mathrm{d}\left(\mathbf{x}, \mathbf{x}^{\prime}\right)<t\right\}\right|
$$

and $B(s)=B(0, s)$, so that $M=\int_{0}^{\pi} d B(x)$. Given a family of codes, we call the function $b(x)$ its distance profile if

$$
b(x)=\lim _{\substack{n \rightarrow \infty \\ \epsilon \rightarrow 0}}(1 / n) \ln B(x-\epsilon, x+\epsilon)
$$

assuming that the limit exists. Throughout this and the next section we use the notation $\theta_{s}=\theta_{s}(R):=$ $\arcsin e^{-R}$.

The decoding mapping for $C$ is defined as follows: $\psi_{\tau}(\mathbf{y})=\mathbf{x}$ if $\mathrm{d}\left(\mathbf{y}, \mathbf{x}^{\prime}\right)-\mathrm{d}(\mathbf{y}, \mathbf{x}) \geq 2 \tau$ for all $\mathbf{x}^{\prime} \in C, \mathbf{x}^{\prime} \neq \mathbf{x}$. If such code vector $\mathbf{x}$ does not exist, decoding results in an erasure. Assume that the transmitted vector $\mathbf{x}$ is displaced by a noise vector $\mathbf{z}$ whose coordinates are i.i.d. Gaussian random variables with mean 0 and unit variance. Let $E_{e}(R, A, \tau)$ and $E_{x}(R, A, \tau)$ be the best attainable exponents of the error and erasure rate, respectively. When $\tau=0$, this is the usual complete decoding, and $E_{e}$ is the reliability function of the Gaussian channel. In this case we will omit 0 from our notation and write $\psi, E(R, A)$. The following lower bound on $E(R, A)$ is classical [17]: let $\theta=\theta_{s}(R)$, then $E(R, A) \geq E_{0}(\theta, A)$, where

$$
E_{0}(\theta, A)= \begin{cases}\frac{A}{4}(1-\cos \theta), & \frac{\pi}{2} \geq \theta \geq \theta_{e} \\ \frac{A}{4}\left(1-\cos \theta_{e}\right)+\ln \frac{\sin \theta}{\sin \theta_{e}}, & \theta_{e} \geq \theta \geq \theta_{c} \\ E_{s p}(\theta, A), & \theta_{c} \geq \theta \geq \operatorname{arccot} \sqrt{A}\end{cases}
$$

where $\csc ^{2} \theta_{e}=\frac{1}{2}+\frac{1}{2} \sqrt{1+\frac{A^{2}}{4}}, \csc ^{2} \theta_{c}=\frac{1}{2}+\frac{A}{4}+\frac{1}{2} \sqrt{1+\frac{A^{2}}{4}}$

$$
\begin{aligned}
E_{s p}(\phi, A) & :=\frac{A}{2}-\frac{\sqrt{A}}{2} g(\phi) \cos \phi-\ln (g(\phi) \sin \phi), \\
g(\phi, A) & :=\frac{1}{2}\left(\sqrt{A} \cos \phi+\sqrt{A \cos ^{2} \phi+4}\right) .
\end{aligned}
$$

This bound will follow as a special case of our derivation.

The (Shannon) volume, or sphere packing bound [17] establishes the existence of codes of rate $R$ with distance arbitrarily close to $\theta_{s}(R)$. It is also straightforward to prove that there exists a code $\mathcal{C}$ of rate $R$ with distance $\theta_{s}$ and distance distribution

$$
B(s) \leq p(n) e^{n(R+\ln \sin \theta)} \quad\left(\theta_{s} \leq \theta \leq \pi-\theta_{s}\right),
$$

where $p(n)$ is some polynomial function. This distribution is induced by the (normalized) uniform measure on $S^{n-1}$ and therefore plays the role analogous to that of the binomial distribution in the Hamming space. The distance profile corresponding to it is $\beta(R, \theta)=R+\sin \theta$. We will examine the behavior of the error rate with decoding $\psi_{\tau}$ being applied to sequences of $\operatorname{codes} \mathcal{C}$ with these properties.

Below we track only one of the two cases, the error-only event, and state results for the erasure rate sparing the reader the detailed analysis. Our goal will be to establish the following theorem.

Theorem 5 Let $R$ be the code rate, let $\theta_{s}=\theta_{s}(R)$ be the code distance and let $\tau>0$. The exponent $E_{e}(R, A, \tau)$ is bounded below by $M(R)$, where for $\pi / 2 \geq \theta_{s}>\theta_{1}$

$$
M(R)=\frac{A}{4}\left(1-\cos \left(\theta_{s}+\tau\right)\right)-G\left(\theta_{s}, \tau\right),
$$


for $\theta_{1} \geq \theta_{s}>\theta_{2}$

$$
M(R)=\frac{A}{4}\left(1-\cos \left(\theta_{1}+\tau\right)\right)+\ln \frac{\sin \theta_{s}(R)}{\sin \theta_{1}}-G\left(\theta_{1}, \tau\right)
$$

and for $\theta_{2}>\theta_{s}$

$$
M(R)=E_{s p}(\rho, A)
$$

Here

$$
G(\phi, \tau)=\frac{1}{2} \ln \left[1+\frac{A \cos ^{2} \frac{\phi+\tau}{2}\left(\sin ^{2} \frac{\phi+\tau}{2}-\sin ^{2}\left(\frac{\phi}{2}+\tau\right)\right)}{\cos ^{2}\left(\frac{\phi}{2}+\tau\right)}\right]
$$

$\theta(x)$ is the (real, positive-valued) function defined implicitly by the equation

$$
\cot \theta=\frac{\cos ^{2} x \tan \left(\frac{\theta}{2}+\tau\right)}{\cos (\theta+2 \tau)-\cos 2 x} \quad(0 \leq x \leq \pi / 2),
$$

$\rho=\rho(R) \in\left[t_{s}, 2 t_{s}\right]$ is the unique angle such that

$$
R+\ln \sin \theta(\rho)+\frac{1}{2} \ln \left(1-\frac{\tan ^{2}((\theta(\rho) / 2)+\tau)}{\tan ^{2} \rho}\right)=0,
$$

$\theta_{1}$ is the root of

$$
\frac{d}{d x}\left(\ln \sin x+\frac{A}{4} \cos (x+\tau)+G(x, \tau)\right)=0
$$

and $\theta_{2}=\theta_{s}\left(R^{*}\right)$, where $R^{*}$ is the root of $\theta(\rho(R))=\theta_{1}$.

A lower bound on the exponent $E_{x}(R, A, \tau)$ is obtained on replacing $\tau$ by $-\tau$ throughout.

Although it is not immediately seen, for $\tau=0$ we have $M(R)=E_{0}(R)$, so in this case the bounds simplify significantly. For instance, $G(\phi, 0)=0$, and the bound (10) reduces to (9), the value $\rho$ equals $\theta_{s}(R)$, the angle $\theta_{1}$ is simply $\theta_{e}$ of $(9 \mathrm{a}-\mathrm{b})$, and so on. We explain these and indicate further connections with bound $(9)$ in remark 6 below. Note that though there seems to be no closed-form expression for the exponents, it is easy to compute them for any given $A, \tau$. It helps to observe that on substituting $\theta(\rho)$ into (15), this equation contains only one unknown, $\rho$. We show the behavior of the bounds in Fig. 2] Note that $M(\rho)>0$ for $0 \leq \rho<\operatorname{arccot} \sqrt{A}$. Note also that $G(\phi, \tau)$ is negative (and usually small), so on omitting it from expressions (10), (11) we still obtain valid lower bounds.

The remaining part of this section is devoted to the proof of this theorem. We begin with some notation and technical results. Let $\operatorname{Con}(\mathbf{x}, \phi)$ denote the circular cone with apex at the origin, axis given by a vector $\mathbf{x} \in \mathbb{R}^{n}$ and solid half-angle $\phi$. We write $f(n) \cong g(n)$ if $\lim _{n \rightarrow \infty} \frac{1}{n} \ln \frac{f(n)}{g(n)}=0$.

We will need the following lemmas.

Lemma 6 [17] Let $\mathbf{x} \in X$ and $\mathbf{z}$ a random Gaussian vector. Let $Q(\phi)$ be the probability that $\mathbf{x}+\mathbf{z} \notin$ $\operatorname{Con}(\mathbf{x}, \phi)$. Then $Q(\phi) \cong e^{-n E_{s p}(\phi, A)}$ and $-d Q(\phi) \cong e^{-n E_{s p}(\phi, A)} d \phi$.

Lemma 7 [17] Let $T(\phi)$ be the area of the spherical cap on the sphere $S^{n-1}(r)$ cut out by a cone Con $(\mathbf{x}, \phi)$. Then as $n \rightarrow \infty$

$$
T(\phi) \sim \frac{2 \pi^{(n-1) / 2} r^{n-1} \sin ^{n-1} \phi}{(n-1) \Gamma\left(\frac{n-1}{2}\right) \cos \phi} .
$$

For the normalized area $\Omega(\phi)=T(\phi) / T(\pi)$ we have

$$
\Omega(\phi) \cong(\sin \phi)^{n}
$$




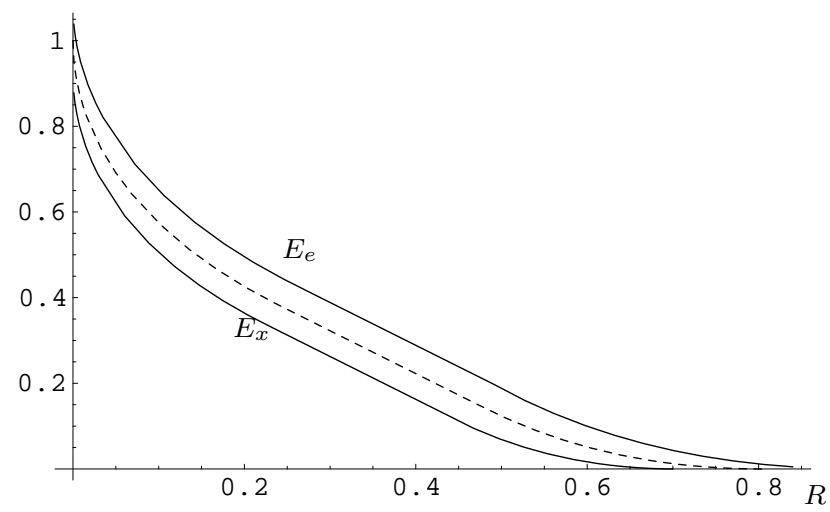

Figure 2: Error bounds from Theorem $5\left(A=4, \tau=0.04 \approx 2.3^{\circ}\right)$. The dashed line is the function $E_{0}(R, A)$.

Lemma 8 (e.g. [6 p.65]) (Laplace method) Let

$$
h(\lambda)=\int_{a}^{b} e^{\lambda q(x)} d x \quad(-\infty \leq a<0<b \leq \infty) .
$$

Suppose that the integral converges absolutely at least for sufficiently large $\lambda$.

(i) Suppose that the absolute maximum of $q(x)$ in $[a, b]$ is attained for $x=0$, that $q^{\prime}(0)$ exists and is continuous in some neighborhood of 0 , and that $q^{\prime \prime}(0)<0$. Then

$$
h(\lambda) \sim \sqrt{-\frac{2 \pi}{\lambda q^{\prime \prime}(0)}} e^{\lambda q(0)} \quad(\lambda \rightarrow \infty) .
$$

(ii) Suppose that $a=0$ and that the absolute maximum of $q(x)$ in $[a, b]$ is attained for $x=0$. Then

$$
h(\lambda) \sim-\frac{e^{\lambda q(0)}}{\lambda q^{\prime}(0)} \quad(\lambda \rightarrow \infty)
$$

provided that $q^{\prime}(0)<0$.

Shannon's approach to bounding the rate of error events is as follows. Let $\mathcal{E}$ denote one of the two events: error, or error or erasure.

Lemma 9 [17] Let $\mathbf{z}$ be the channel error vector. Then

$$
P(\mathcal{E}, C) \leq \min _{\rho} P(\mathcal{E} \mid w(\mathbf{z}) \leq \rho)+P(w(\mathbf{z}) \geq \rho) .
$$

Generally, the minimum is attained for different $\rho$ depending on the meaning of $\mathcal{E}$. Note that to obtain a valid bound we do not have to optimize on $\rho$, taking an arbitrary value at our convenience. Below we always assume that $\rho<90^{\circ}$. 

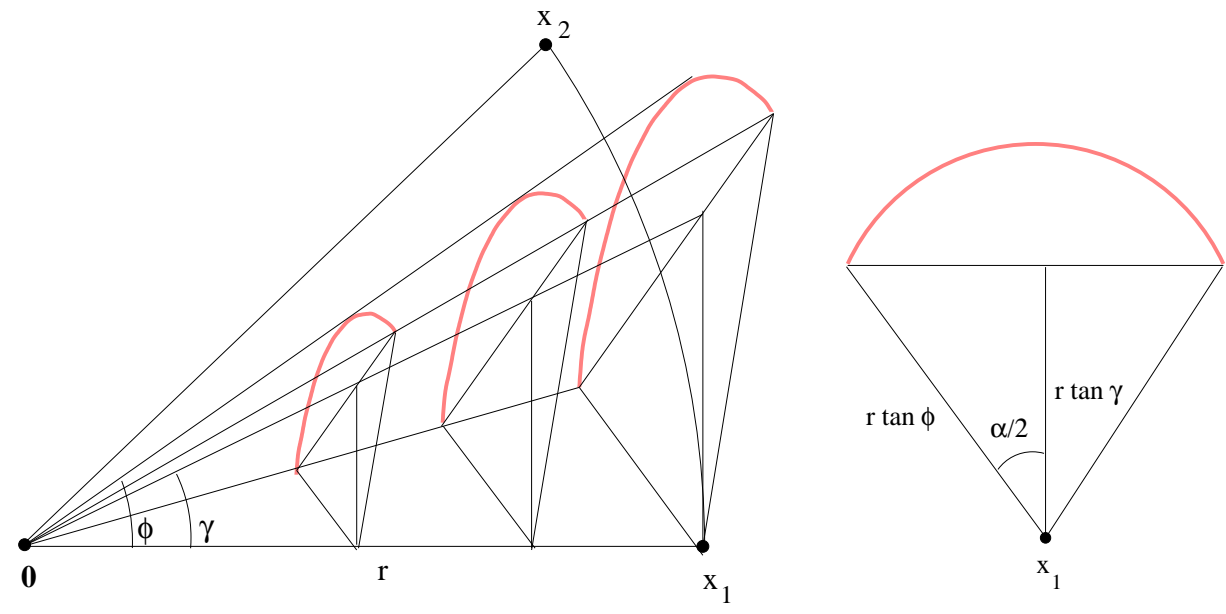

Figure 3: Derivation of Lemma10

Lemma 10 Let $\mathbf{x}_{1} \in C$ be transmitted and let $P_{\theta}\left(\mathbf{x}_{1} \rightarrow \mathbf{x}_{2}\right)$ be the probability that decoding $\psi_{\tau}$ mistakes $\mathbf{x}_{1}$ for a fixed code vector $\mathbf{x}_{2}$ with $d\left(\mathbf{x}_{1}, \mathbf{x}_{2}\right)=\theta$. Then $P_{\theta}\left(\mathbf{x}_{1} \rightarrow \mathbf{x}_{2}\right) \cong F(\theta, \tau)$, where

$$
F(\theta, \tau):=\int_{\theta / 2+\tau}^{\rho}\left(1-\frac{\tan ^{2}(\theta / 2+\tau)}{\tan ^{2} \phi}\right)^{n / 2} e^{-n E_{s p}(\phi, A)} d \phi .
$$

PROOF. Let $\mathbf{z}$ be the error vector with $w(\mathbf{z})=\phi,\|\mathbf{z}\|=r$. Let us compute the fraction of such errors that lead to a decoding error that outputs $\mathbf{x}_{2}$. For this to happen it suffices that $\phi \geq \theta / 2+\tau$ and $\left\|\mathbf{x}_{2}-\mathbf{y}\right\| \leq$ $\left\|\mathbf{x}_{1}-\mathbf{y}\right\|$, where $\mathbf{y}=\mathbf{x}_{1}+\mathbf{z}$ is the received vector. This fraction equals the normalized area of the spherical cap cut out on the surface of $\operatorname{Con}(\mathbf{x}, \phi)$ by the hyperplane perpendicular to $\mathbf{x}_{1}$ and located at a distance $r$ from the origin. Taking in Fig. $3 \gamma=\theta / 2+\tau$, we compute for the angle $\alpha$ of this cap

$$
\sin ^{2} \alpha / 2=1-\left(\frac{r \tan (\theta / 2+\tau)}{r \tan \phi}\right)^{2}=1-\frac{\tan ^{2}(\theta / 2+\tau)}{\tan ^{2} \phi}
$$

The normalized area $\Omega$ of the cap in question is given by Lemma 7

$$
\Omega \cong(\sin \alpha / 2)^{n}
$$

and does not depend on the distance $r$ from the origin. Hence we may integrate $r$ out and obtain for the differential probability

$$
P\left(\mathbf{x}_{1} \rightarrow \mathbf{x}_{2} \mid \phi \leq w(\mathbf{z}) \leq \phi+d \phi\right) \cong-\left(1-\frac{\tan ^{2}(\theta / 2+\tau)}{\tan ^{2} \phi}\right)^{n / 2} d Q(\phi)
$$

Now the claim of the lemma is obtained by integrating on $\phi$ from $\theta / 2+\tau$ (because errors of smaller weight cannot lead to decoding error) to $\rho$ (because errors of greater weight are assumed to always lead to a decoding error) and substituting $d Q(\phi)$ from Lemma 6

Putting the pieces together, we obtain the following bound on the probability of error for spherical codes under $\psi_{\tau}$. 
Theorem 11 Let $C$ be a code of rate $R$ and distance $\theta$ with distance profile $b(\theta)$. Then for any $\rho \in$ $(\theta / 2, \pi / 2)$

$$
P(\mathcal{E} ; C) \lesssim \int_{\theta}^{2(\rho-\tau)} e^{n b(\theta)} F(\theta, \tau) d \theta+Q(\rho) .
$$

PROOF. Follows on applying Lemmas 9 and 10 and the union bound.

Now we are ready to complete the proof of Theorem 5. First let us find the asymptotic behavior of $F(\theta, \tau)$. We have $F(\theta, \tau) \cong \int e^{n q(\phi)} d \phi$, where

$$
q(\phi)=\frac{1}{2} \ln \left(1-\frac{\tan ^{2}(\theta / 2+\tau)}{\tan ^{2} \phi}\right)-E_{s p}(\phi, A) .
$$

From the equation $q^{\prime}(\phi)=0$ we find that the maximum of the integrand is attained for $\phi_{0}(\tau)$ defined by

$$
\sin ^{2} \phi_{0}=\frac{4+A \sin ^{2}(\theta+2 \tau)}{2(2+A+A \cos (\theta+2 \tau))} .
$$

The asymptotic value of the integral is obtained by Lemma 8 and depends on the location of $\phi_{0}$ with respect to the integration limits. First, it is easy to see that $\phi_{0}>\theta / 2+\tau$ for any $0<A<\infty, 0<\theta<\pi-2 \tau$. Indeed, it suffices to show that $\sin ^{2} \phi_{0}>\sin ^{2}(\theta / 2+\tau)$. Therefore, compute

$$
\begin{aligned}
2(2+A+ & A \cos (\theta+2 \tau))\left(\sin ^{2} \phi_{0}-\sin ^{2}\left(\frac{\theta}{2}+\tau\right)\right) \\
= & 4+A \sin ^{2}(\theta+2 \tau)-2 \sin ^{2}\left(\frac{\theta}{2}+\tau\right)(2+A+A \cos (\theta+2 \tau)) \\
= & 4\left(1-\sin ^{2}\left(\frac{\theta}{2}+\tau\right)\right)>0 .
\end{aligned}
$$

It remains to examine the location of $\phi_{0}$ with respect to the upper limit of integration, $\rho$. We have the two following cases.

1. $\phi_{0}<\rho$. Then by Lemma 8 (i) the behavior of the integral is determined by $\phi$ in the neighborhood of $\phi_{0}$. We obtain

$$
n^{-1} \ln F(\theta, \tau) \sim \frac{1}{2} \ln \left(1-\frac{\tan ^{2}(\theta / 2+\tau)}{\tan ^{2} \phi_{0}}\right)-E_{s p}\left(\phi_{0}, A\right) .
$$

Next let us proceed to computing the asymptotic expression for the outer integral in (17). Denoting the integrand by $D$, substituting the value of $\phi_{0}$ and taking $b(\theta)=\beta(R, \theta)$, after all simplifications, we arrive at the expression

$$
-n^{-1} \ln D \sim \frac{A}{4}(1-\cos (\theta+\tau))-\beta(R, \theta)-G(\theta, \tau) .
$$

Now invoke again Lemma 8 . The main term of the integral depends on the relative location of the maximizing value of $\theta$, denoted by $\theta_{1}$, and the integration limits. As it turns out, for $\theta_{1}$ we have $0<\theta_{1}<2 \rho-2 \tau$, so what matters is the mutual location of $\theta_{1}$ and $\theta_{s}$. If $\theta_{1}<\theta_{s}$, then by Lemma 8 (ii) the main term is determined by $\theta=\theta_{s}$. Since $\beta\left(R, \theta_{s}\right)=0$, we obtain for $E_{e}(R, A, \tau)$ the bound

$$
E_{e}(R, A, \tau) \geq \frac{A}{4}\left(1-\cos \left(\theta_{s}+\tau\right)\right)-G\left(\theta_{s}, \tau\right),
$$

which is (10). On the other hand, if $\theta_{1} \geq \theta_{s}$, then we use part (i) of the same lemma and obtain the bound (11). This proves the first two parts of the theorem except the upper limit $\theta_{2}$ of range of angles in (11) which will be established later. 
2. $\phi_{0} \geq \rho$. Now the asymptotic value of $F(\theta, \tau)$ is determined by $\phi=\rho$, and so

$$
n^{-1} \ln F(\theta, \tau) \sim \frac{1}{2} \ln \left(1-\frac{\tan ^{2}(\theta / 2+\tau)}{\tan ^{2} \rho}\right)-E_{s p}(\rho, A) .
$$

We proceed to computing the asymptotic expression for the outer integral in (17). Again taking $b(\theta)=$ $\beta(R, \theta)$ and denoting the integrand by $D$, we obtain

$$
n^{-1} \ln D \sim R+\ln \sin \theta+\frac{1}{2} \ln \left(1-\frac{\tan ^{2}(\theta / 2+\tau)}{\tan ^{2} \rho}\right)-E_{s p}(\rho, A) .
$$

Differentiating, we find that the maximum of this expression on $\theta$ is attained for $\theta=\theta(\rho)$, and it is possible to prove that with our choice of $\rho$ the value $\theta(\rho)$ is always within the integration range: $\theta_{s} \leq \theta(\rho)<2(\rho-\tau)$. Furthermore, by (15) the first three terms in the expression for $D$ add up to zero. Concluding, in this case the integral on $\theta$ evaluates asymptotically to

$$
\int_{\theta_{s}}^{2 \rho-2 \tau} e^{n(\beta(R, \theta)+\ln F(\theta, \tau))} d \theta \cong e^{-n E_{s p}(\rho, A)} .
$$

Clearly, the second term in (17) has the same asymptotic behavior, which is therefore the answer in the case studied. It remains to find the value $\theta_{2}$ when the main term of the estimate moves from (11) to (12). This obviously happens when the two functions first become equal as the angle $\theta_{s}$ decreases from $\theta_{1}$ or when the rate $R$ reaches the value such that $\theta(\rho(R))=\theta_{1}$, or when $\theta_{s}=\theta_{2}$. This concludes the proof of (12) and thus of the theorem.

REMARK 6: The results of [17] are obtained from this theorem by substituting $\tau=0$ in (14). Denoting $\theta(\rho)$ in this case by $\theta_{E}$, we find that $\cos \theta_{E}=\cos ^{2} \rho$. Further, substituting $\theta_{E}$ into 15), we find that $\rho(R)=\theta_{s}(R)$, i.e., the optimizing value of the decoding radius in (9) in this case is $\theta_{s}$. Taking $\tau=0$ in (16), we obtain for $\theta_{1}$ the explicit equation $\cos \theta_{1}=(A / 4) \sin ^{2} \theta_{1}$ whence $\theta_{1}=\theta_{e}$. Further, the equation for $R^{*}$ which in general is $\theta(\rho(R))=\theta_{1}$, now reduces to $\theta_{E}=\theta_{1}$ or

$$
\csc ^{2} \theta_{E}=\left[\sin ^{2} \theta_{s}\left(R^{*}\right)\left(2-\sin ^{2} \theta_{s}\left(R^{*}\right)\right)\right]^{-1}=\frac{1}{2}\left(1+\sqrt{1+\frac{A^{2}}{4}}\right) .
$$

From this we find $R^{*}=-\ln \sin \theta_{c}$, or $\theta_{2}=\theta_{c}$ of $(9 \mathrm{~b}-\mathrm{c})$. Hence $\theta_{2}$ equals the critical angle and $R^{*}$ equals the critical rate of the channel.

These remarks also enable us to make some observations on typical error events in the course of decoding of $\operatorname{codes} \mathcal{C}$. They are easier understood for $\tau=0$. Since the codes $\mathcal{C}$ generally are not distance invariant, the following is valid on average only.

1. Suppose that $\pi / 2>\theta_{s}>\theta_{e}$, then the errors that contribute to the main term of $E(R, A)$ most probably are of weight $\phi_{0} \in\left(\theta_{s} / 2, \theta_{s}\right)$. In the case of decoding error the typical distance of the output code vector from the transmitted one equals $\theta_{s}$ and does not depend on the level of noise in the channel.

2. For $\theta_{e}>\theta_{s}>\theta_{c}$, typical errors are also of weight $\phi_{0}$ and result into code vectors at distance $\theta_{e}$ from the transmitted one. From the moment that $\theta_{E}=\theta_{e}$ or $\theta_{c}=\theta_{s}$, typical errors are of weight $\theta_{s}$ and the resulting code vectors are distance $\theta_{E}$ from the transmitted one. The error rate in this case does not depend on the actual channel noise or on the distance of the code. 
REMARK 7: (The Elias angle). Note that the value $\theta_{E}$ gives the answer to the following geometric question. Let $\mathbf{x} \in \mathcal{C}$ be a vector in a code of rate $R$ and distance $\theta_{s}$. Consider all the neighbors $\mathbf{x}^{\prime}$ of $\mathbf{x}$ in $\mathcal{C}$ such that $\mathrm{d}\left(\mathbf{x}, \mathbf{x}^{\prime}\right)=\alpha$ for a given $\alpha$ and draw the cones $\operatorname{Con}\left(\mathbf{x}^{\prime}, \theta_{s}\right)$ about them. What is the minimum value of $\alpha$ such that fraction of the surface of $\operatorname{Con}\left(\mathbf{x}, \theta_{s}\right)$ covered by these cones asymptotically becomes one? The answer follows from (15) and is given by $\alpha=\theta_{E}$. This parameter plays the same role for $S^{n-1}$ as the Elias radius for the Hamming space (see, e.g., [1]), therefore we call it the Elias angle. This also hints, by the same geometric argument as in the Hamming case at the bound $\theta \leq \theta_{E}$ for the maximal attainable minimum distance of a spherical code of rate $R$. Solving the first inequality in (19) for $R$, we obtain a different form of this bound, namely $R \leq-\ln (\sqrt{2} \sin (\theta / 2))$. This is an old bound of Rankin [15] and Coxeter [4] on the rate of a spherical code of distance $\theta$ whose proof we therefore obtain.

REMARK 8: Note that though we emphasized codes $\mathcal{C}$ in our derivation, many parts of it, such as bound (17), apply to any sequence of codes with a known distance profile. They are also applicable to binary codes used over the binary-input Gaussian channel. Let $C$ be a binary spherical code, i.e. a subset of $S^{n-1}(\sqrt{A n})$ such that coordinates of every vector in $C$ take values $\pm \sqrt{A}$. Let $d(C)$ and $\theta(C)$ be the minimum Hamming and angular distance in $C$ respectively, then $d(C)=n(1-\cos \theta(C)) / 2$. We can specialize bound (17) to this case as follows:

$$
P(\mathcal{E}, C) \leq \sum_{w=d(C)}^{\lfloor n(1-\cos 2 \rho) / 2\rfloor} A_{w} F\left(\theta_{w}, \tau\right)+Q(\rho),
$$

where $\left(A_{d}, \ldots, A_{n}\right)$ is the distribution vector of Hamming distances in $C$ and $\theta_{w}=\arccos (1-2 w / n)$. It is straightforward to compute the trade-off bounds analogous to Theorem 5 For $\tau=0$ they reduce to a bound on the error rate of complete decoding for $C$ which can be used for finite length as well. For that purpose, more accurate approximations on $F(\theta, \tau)$ than those used above are readily available. In particular, the normalized area of the spherical cap can be computed with arbitrary precision from the asymptotic series provided by the Laplace method [12], and a more precise expression for $Q(\phi)$ than the one quoted in Lemma 6 is given in [17, Eq. (51)]. Asymptotically (20) becomes the same as Poltyrev's "tangential-sphere" bound [13]; for binary linear codes with binomial weight spectrum $\mathcal{A}_{i}$ (see Sect. 2) we immediately recover the part of the random coding exponent below the cutoff rate.

\section{Related results: Bounded distance decoding and error detection}

Let us address a related question, that of error exponents for bounded distance decoding of spherical codes. Consider the following partial decoding mapping $\tilde{\psi}_{\tau}: X \rightarrow C:$ if $\mathbf{y}$ is within distance $\tau$ of a code vector $\mathbf{x}$, then $\tilde{\psi}_{\tau}(\mathbf{y})=\mathbf{x}$, and if there is not such $\mathbf{x}$, the value of $\tilde{\psi}_{\tau}(\mathbf{y})$ is undefined. Recall that by distance $\mathrm{d}(\mathbf{x}, \mathbf{y})$ we mean the angle $\angle(\mathbf{x}, \mathbf{y})$. Again we are interested in the best attainable error exponent of such decoding for spherical codes. The following proposition is obvious if in Lemma 9 we take $\rho=\pi / 2$, and use Lemmas 10 and 6

Proposition 12 Let $C$ be a code in $X$ with distance $\theta(C)>0$ and distance profile $b(\theta)$. Then for any $\epsilon>0$ the probability of decoding error

$$
P_{d e}(C) \lesssim \iint e^{n\left(b(\theta)+\frac{1}{2} \ln \left(1-\frac{\tan ^{2}(\theta-\tau)}{\tan ^{2} \phi}\right)\right)} d Q(\phi) d \theta+e^{-n E_{s p}(\pi / 2-\tau-\epsilon, A)},
$$

where $\theta(C) \leq \theta \leq \pi / 2-\tau-\epsilon$ and $\max (\theta(C) / 2, \theta-\tau) \leq \phi \leq \theta+\tau$. 
Note that the choice of the upper limit $\theta<\pi / 2-\tau-\epsilon$ is forced by Lemma 10

Let us first study the asymptotic behavior of the integral on $\phi$. Letting $\tilde{F}(\theta, \tau)=\int e^{n q(\phi)} d \phi$ with

$$
q(\phi)=\frac{1}{2} \ln \left(1-\frac{\tan ^{2}(\theta-\tau)}{\tan ^{2} \phi}\right)-E_{s p}(\phi, A)
$$

we find the root $\phi_{0}$ of $q^{\prime}(\phi)=0$ to satisfy

$$
\sin ^{2} \phi_{0}=\frac{4+A \sin ^{2}(2(\theta-\tau))}{4+2 A+2 A \cos (2(\theta-\tau))}
$$

(cf. (18)). It is easy to see that $\phi_{0}<\pi / 2$. By the calculation following (18) we know that also in the present situation $\phi_{0}>\theta-\tau$, so the asymptotics of $\tilde{F}(\theta, \tau)$ depends on the mutual location of $\phi_{0}$ and $\theta+\tau$. Thus we obtain for the error exponent $\tilde{E}_{e}(R, A, \tau)=n^{-1} \ln P_{d e}(C)$

$$
\tilde{E}_{e}(R, A, \tau) \gtrsim \max _{\theta(C) \leq \theta \leq \pi / 2-\tau}\left(-b(\theta)-q\left(\theta_{0}\right)\right)
$$

where $\theta_{0}=\phi_{0}$ if $\phi_{0}<\theta+\tau$ or $\theta_{0}=\theta+\tau$ otherwise. The first situation usually occurs for high code rates, and the last for low rates. As above, the second term in (21) can improve the high-rate case.

We conclude this section with studying error detection with spherical codes. Generally error detection proceeds as follows: if the received vector $\mathbf{y}$ is contained in $C$, the decoder outputs $\mathbf{y}$, otherwise its output is undefined. Clearly for any finite-size code $C \subset S^{n-1}$ the probability of undetected error is zero, therefore we define error detection as a limiting case of bounded distance decoding and study the behavior of $P$ as $\tau \rightarrow 0$. Since the code is a finite set, the cumulative measure of spherical caps about code vectors tends to zero if so does their angle. Hence the error probability $P_{d e}(C)$ is determined by the decrease rate of the area of a spherical cap. Assume that $C$ is a code with distance $\theta$ separated from 0 and distance profile $b(\theta)$. If $\tau=0$, then by 18 we have

$$
\sin ^{2} \phi_{0}-\sin ^{2} \theta=4\left(1-\sin ^{2} \theta\right)>0
$$

so by continuity for small positive $\tau$ also $\sin ^{2} \phi_{0}>\sin ^{2}(\theta+\tau)$. Hence we obtain

$$
n^{-1} \ln \tilde{F}(\theta, \tau) \sim \frac{1}{2} \ln \left(1-\frac{\tan ^{2}(\theta-\tau)}{\tan ^{2}(\theta+\tau)}\right)-E_{s p}(\theta+\tau, A) .
$$

Since for $\tau \rightarrow 0$

$$
\frac{\tan ^{2}(\theta-\tau)}{\tan ^{2}(\theta+\tau)}=1-\frac{8}{\sin 2 \theta} \tau+O\left(\tau^{2}\right)
$$

we conclude that the probability of undetected error essentially does not depend on the distance profile of $C$ and behaves as

$$
P_{u e} \cong \exp (n \ln \sqrt{8 \tau \csc 2 \theta(C))})=(8 \tau \csc 2 \theta(C))^{n / 2} .
$$

We see that basically one and the same behavior can be claimed for any code with minimum distance $\theta$ separated from 0; thus the asymptotic answer for the undetected error rate of spherical codes is known exactly (unlike the more difficult Hamming case where it essentially depends on optimal codes).

Acknowledgment. Thanks to an anonymous referee for pointing out a potential error in the original derivation.

\section{Appendix}


A PROOF OF THEOREM 2 Let $\mathcal{C}$ be a binary linear code of rate $R$, distance $d$ and weight distribution $A_{i}(\mathcal{C})$, where $A_{i}(\mathcal{C}) \cong \mathcal{A}_{i}$. The weight profile of $\mathcal{C}$ has the form $\alpha_{0}(\omega):=h(\omega)-h\left(\delta_{\mathrm{GV}}\right)$. Let $F_{+}$denote the undetected error event and $F_{-}$the error-or-erasure event. Assume w.l.o.g. that the transmitted vector is all-zero and that $\mathbf{e}$ is the channel error vector. The probability of the error events can be bounded above as follows:

$$
P\left(F_{ \pm}\right) \leq P\left(F_{ \pm} \mid \mathbf{e} \in S_{r^{ \pm}}(0)\right)+P\left(\mathbf{e} \notin S_{r^{ \pm}}(0)\right)
$$

for some positive $r^{+}$and $r^{-}$. Below we choose $r^{ \pm}=d \pm 2 t$. More concretely, we have

$$
P\left(F_{ \pm} \mid \mathbf{e} \in S_{r^{ \pm}}(0)\right) \leq \sum_{w=d}^{2 r^{ \pm} \mp 2 t} A_{w}(\mathcal{C}) \sum_{e=w / 2 \pm t}^{r^{ \pm}} p^{e}(1-p)^{n-e} \sum_{s=0}^{e \mp 2 t} p_{e, s}^{w}
$$

More accurately, the range of the summation index $e$ in the above expression is $w \geq\lceil w / 2\rceil \pm t$ if $w$ is odd and $w / 2+1 \pm t$ if $w$ is even; we will ignore this. Let us proceed with the undetected error case and rewrite the estimate in an explicit form, substituting the value of $A_{w}$ :

$$
\begin{gathered}
P\left(F_{+}\right) \leq 2^{-n(1-R)} \sum_{w=d}^{2 d+2 t}\left(\begin{array}{c}
n \\
w
\end{array}\right) \sum_{e=w / 2+t}^{d+2 t} p^{e}(1-p)^{n-e} \sum_{i=\left\lceil\frac{w}{2}\right\rceil+t}^{e}\left(\begin{array}{c}
w \\
i
\end{array}\right)\left(\begin{array}{c}
n-w \\
e-i
\end{array}\right) \\
+\sum_{e=d+2 t+1}^{n}\left(\begin{array}{c}
n \\
e
\end{array}\right) p^{e}(1-p)^{n-e} .
\end{gathered}
$$

To facilitate transition to this expression from (22) notice that if $\mathbf{c}$ is the incorrect codeword of weight $w>0$ output by the decoder and $\mathbf{e}$ is the error vector then the index $i=|\operatorname{supp}(\mathbf{e}) \cap \operatorname{supp}(\mathbf{c})|$.

The product $\left(\begin{array}{c}w \\ i\end{array}\right)\left(\begin{array}{c}n-w \\ e-i\end{array}\right)$ is maximized for

$$
i \approx \frac{e w}{n} \leq \frac{(d+2 t) w}{n} \leq \frac{w}{2}+t
$$

where the last step follows (for large $n$ ) by the assumption of the theorem $R \geq 1-h(1 / 2-\tau)$ which translates into $\delta_{\mathrm{GV}}(R)+\tau \leq 1 / 2$. Therefore the sum on $w$ in (23) for large $n$ can be estimated from above by

$$
\begin{aligned}
& n \sum_{e=d / 2+t}^{d+2 t} p^{e}(1-p)^{n-e} \sum_{w=d}^{2(e-t)}\left(\begin{array}{l}
n \\
w
\end{array}\right)\left(\begin{array}{c}
w \\
w / 2+t
\end{array}\right)\left(\begin{array}{c}
n-w \\
e-w / 2-t
\end{array}\right) \\
& \cong \sum_{e}\left(\begin{array}{l}
n \\
e
\end{array}\right) p^{e}(1-p)^{n-e} \sum_{w}\left(\begin{array}{c}
e \\
w / 2+t
\end{array}\right)\left(\begin{array}{c}
n-e \\
w / 2-t
\end{array}\right)
\end{aligned}
$$

( since $\left.\left(\begin{array}{l}n \\ w\end{array}\right) p_{e-2 t, e}^{w}=\left(\begin{array}{l}n \\ e\end{array}\right) p_{e-2 t, w}^{e}\right)$. In the sum on $w$ we are counting the number of vectors of weight $w$ which are distance $e-2 t$ away from a given vector of weight $e$. This number is maximized when

$$
\frac{e-t-\frac{w}{2}}{e} \approx \frac{e-2 t}{n}
$$

Introducing the notation $w=\omega n, e=\rho n$, we can rewrite this relation as

$$
\omega^{*}=2 \rho(1-\rho)-2 \tau(1-2 \rho) .
$$


Thus the expression in (25) is $\cong$-equivalent to

$$
\begin{aligned}
\sum_{e}\left(\begin{array}{l}
n \\
e
\end{array}\right) p^{e}(1-p)^{n-e}\left(\begin{array}{c}
e \\
\rho(n-e)+2 t \rho
\end{array}\right)\left(\begin{array}{c}
n-e \\
(\rho-2 \tau)(n-e)
\end{array}\right) \\
\cong \sum_{e}\left(\begin{array}{c}
n \\
e
\end{array}\right)\left(\begin{array}{c}
n \\
e-2 t
\end{array}\right) p^{e}(1-p)^{n-e} \\
\cong \max _{\delta_{\mathrm{GV}} / 2+\tau \leq \rho \leq \delta_{\mathrm{GV}}+2 \tau} \exp [-n(D(\rho \| p)-h(\rho-2 \tau))] .
\end{aligned}
$$

The last exponent is maximized for $\rho=\rho_{0}^{+}$, and thus the unrestricted maximum on $\omega$ is attained for $\omega=\omega_{0}$. The cases (a)-(c) of the theorem are realized depending on how these values are located with respect to the optimization limits

$$
\omega \geq \delta, \quad \delta_{\mathrm{GV}} / 2+\tau \leq \rho \leq \delta_{\mathrm{GV}}+2 \tau .
$$

If both $\rho_{0}$ and $\omega_{0}$ satisfy these inequalities, we substitute them into (26), recall the factor $2^{R n-n}$ from (23) and arrive at case (b) of the bound $M_{+}$in (6).

If $\rho_{0}^{+}>\delta_{\mathrm{GV}}+2 \tau$ then we substitute $\rho=\delta_{\mathrm{GV}}+2 \tau, \omega=\omega^{*}$ and obtain the expression

$$
D\left(\delta_{\mathrm{GV}}+2 \tau \| p\right)-h\left(\delta_{\mathrm{GV}}\right)+(1-R)=D\left(\delta_{\mathrm{GV}}+2 \tau \| p\right),
$$

i.e., case (c). Finally if $\omega_{0} \leq \delta$, we substitute $w=d$ in (24) and obtain

$$
\begin{aligned}
2^{R n-n} & \sum_{e=d / 2+t}^{d+2 t} p^{e}(1-p)^{n-e}\left(\begin{array}{l}
n \\
d
\end{array}\right)\left(\begin{array}{c}
d \\
d / 2+t
\end{array}\right)\left(\begin{array}{c}
n-d \\
e-d / 2-t
\end{array}\right) \\
& \cong\left(\begin{array}{c}
d \\
d / 2+t
\end{array}\right) \max _{e \geq d / 2+t}\left(\begin{array}{c}
n-d \\
e-d / 2-t
\end{array}\right) p^{e}(1-p)^{n-e} \quad\{a:=e-t\} \\
& =2^{-\nu t}\left(\begin{array}{c}
d \\
d / 2+t
\end{array}\right) \max _{a \geq d / 2}\left(\begin{array}{c}
n-d \\
a-d / 2
\end{array}\right) p^{a}(1-p)^{n-a} .
\end{aligned}
$$

The last maximum is attained for $a-d / 2 \approx(n-d) p$. Substituting and switching to exponents, we arrive at the case (a) in (6).

A proof is needed to show that in this case the first of the two terms in (23) provides the dominating exponent; this is a straightforward calculation which we shall omit. This completes the analysis of the undetected error event $F_{+}$.

Let us sketch the proof in the error-and-erasure case $F_{-}$. Now the sum (22) can be written as

$$
2^{R n-n} \sum_{w=d}^{2 d-2 t}\left(\begin{array}{l}
n \\
w
\end{array}\right) \sum_{e=w / 2-t}^{d-2 t} p^{e}(1-p)^{n-e} \sum_{i=w / 2-t}^{e}\left(\begin{array}{c}
w \\
i
\end{array}\right)\left(\begin{array}{c}
n-w \\
e-i
\end{array}\right) .
$$

We would like to prove that the maximum on $i$ which is again attained for $i \approx e w / n$, at least for large $n$ falls below $w / 2-t$. This will follow from the inequality

$$
\omega \delta-\frac{\omega}{2} \leq(2 \omega-1) \tau
$$

which is proved as follows. We can assume that $\omega<1 / 2$. By assumption, $t \leq p n / 2$ and hence $t \leq d / 2$ since $\delta_{\mathrm{GV}}(R) \geq p$ for $R \leq \mathcal{C}$. Then

$$
\omega \delta-\frac{\omega}{2}+(1-2 \omega) \tau \leq \frac{1}{2}(\delta-\omega) \leq 0 .
$$


Hence for any $\rho \leq \delta-2 \tau$ we have

$$
\omega \rho \leq \omega(\delta-2 \tau) \leq \frac{\omega}{2}-\tau
$$

as desired. So instead of (24) we obtain the expression

$$
n \sum_{e=d / 2-t}^{d-2 t} p^{e}(1-p)^{n-e} \sum_{w=d}^{2(e+t)}\left(\begin{array}{l}
n \\
w
\end{array}\right)\left(\begin{array}{c}
w \\
w / 2-t
\end{array}\right)\left(\begin{array}{c}
n-w \\
e-w / 2+t
\end{array}\right) .
$$

The remaining part of the analysis of this case proceeds as above except that $t$ is replaced by $-t$ throughout. In particular, $\omega^{*}=2 \rho(1-\rho)+2 \tau(1-2 \rho)$, the optimum on $\rho$ is attained for $\rho_{0}^{-}$and so on.

\section{References}

[1] A. Barg and G. D. Forney, Jr., Random codes: Minimum distances and error exponents, IEEE Trans. Inform. Theory 48 (2002), no. 9, 2568-2573.

[2] R. E. Blahut, Principles and practice of information theory, Addison-Wesley, Reading, MA, 1987.

[3] E. L. Blokh and V. V. Zyablov, Linear concatenated codes, Nauka, Moscow, 1982, (In Russian).

[4] H. S. M. Coxeter, An upper bound for the number of equal nonoverlapping spheres that can touch another of the same size, Proc. Symp. Pure Math., vol. 7, Providence: AMS, 1963, pp. 53-72.

[5] I. Csiszár and J. Körner, Information theory. Coding theorems for discrete memoryless channels, Akadémiai Kiadó, Budapest, 1981.

[6] N. G. de Bruijn, Asymptotic methods in analysis, North-Holland, Amsterdam, 1958.

[7] G. D. Forney, Jr., Concantenated codes, MIT Press, Cambridge, MA, 1966.

[8] - Exponential error bounds for erasure, list, and decision feedback schemes, IEEE Trans. Inform. Theory 14 (1968), no. 2, 206-220.

[9] R. G. Gallager, Low-density parity-check codes, MIT Press, Cambridge, MA, 1963.

[10] - Information theory and reliable communication, John Wiley \& Sons, New York e.a., 1968.

[11] V. I. Levenshtein, Bounds on the probability of undetected error, Problemy Peredachi Informatsii 13 (1977), no. 1, 3-18.

[12] F. W. J. Olver, Asymptotics and special functions, A K Peters Ltd., Wellesley, MA, 1997. MR 97i:41001

[13] G. Sh. Poltyrev, Bounds on the decoding error probability of binary linear codes via their spectra, IEEE Trans. Inform. Theory 40 (1994), no. 4, 1284-1292.

[14] - On coding without restrictions for the AWGN channel, IEEE Trans. Inform. Theory 40 (1994), no. 4, 409-417.

[15] R. A. Rankin, The closest packing of spherical caps in $n$ dimensions, Proc. Glasgow Math. Assoc. 2 (1955), 139-144. 
[16] S. Shamai (Shitz) and I. Sason, Variations on the Gallager bounds, connections and applications, IEEE Trans. Inform. Theory (2002), no. 12, 3029-3051.

[17] C. E. Shannon, Probability of error for optimal codes in a Gaussian channel, Bell Syst. Techn. Journ. 38 (1959), no. 3, 611-656.

[18] N. Shulman and M. Feder, Random coding techniques for nonrandom codes, IEEE Trans. Inform. Theory 45 (1999), 2101-2104.

[19] A. J. Viterbi and J. K. Omura, Principles of digital communication and coding, McGraw-Hill, 1979.

[20] A. D. Wyner, Capabilities of bounded discrepancy decoding, Bell Syst. Techn. Journ. (1965), 10611122. 